\title{
A Case of Acute Myocardial Infarction due to Coronary Spasm that Resulted in Thrombi
}

Sadahiro H*, Takamichi M, J unji Y, Takamasa I, Keita W, Yuichiro S, Ryo M, Ryoichi M, Naoyuki M, Masahiro S, Tetsuo Y, Nobuhiro H, Yasutoshi $\mathbf{N}$ and Toshihiro $\mathbf{N}$

Department of Cardiology, J apanese Red Cross

Musashino Hospital, J apan

*Corresponding author: Sadahiro H, Department of Cardiology, J apanese Red Cross Musashino Hospital, 1-26-1 Kyonan-cho, Musashino-City, Tokyo 181-8610, Japan

Received: July 24, 2017; Accepted: August 17, 2017; Published: August 30, 2017

\section{Ablbreviation}

ECG: Electrocardiogram; ACS: Acute Coronary Syndrome; LAD: Left Anterior Descending Artery; CAS: Coronary Arterial Spasm; NO: Nitrogen Monoxide; MDA-LDL: Malondialdehyde-Modified Low Density Lipoprotein; ACh: Acetylcholine

\section{Case Presentation}

An 82-year-old woman with chest pain was transferred to our hospital by ambulance. Electrocardiography (ECG) revealed STsegment depression in leads I, aVL, and V2-6, and positive troponin I expression. We made a diagnosis of acute coronary syndrome (ACS) and performed emergency coronary angiography. The coronary artery was neither tortuous nor sclerosed, but the filling defect hada "pebble" appearance in the distal portion of the left anterior descending (LAD) artery (Figure 1). Left ventriculography revealed hypokinesis of the cardiac apex (Figure 2A, 2B). She sometimes had chest pain at rest, and the culprit lesion was poorly atherosclerotic and distal; thus, we considered that the cause of the ACS was coronary spasm rather than simple plaque rupture. She was discharged from the hospital within 8 days, with no complications. Coagulation abnormalities and atrial fibrillation were not observed in the blood test and ECG monitoring. During the acute phase and after discharge, she received anticoagulation therapy, single antiplatelet therapy, and coronary vasodilator therapy containing a calcium antagonist. One year later, reexamination with coronary angiography was performed. The filling defect in the LAD artery disappeared, and no significant stenosis and dissection were observed. The left ventricular wall motion had improved (Figure 2C, 2D). Acetylcholine intracoronary administration induced coronary arterial spasm (CAS) with symptoms in both coronary arteries (Figure 3). She was diagnosed as having coronary vasospastic angina, was suspected to have caused the ACS.

\section{Discussion}

We suspected thrombi formation due to CAS based on the characteristic angiographic findings in this case and treated it successfully. The coronary artery was smoothly shaped with little

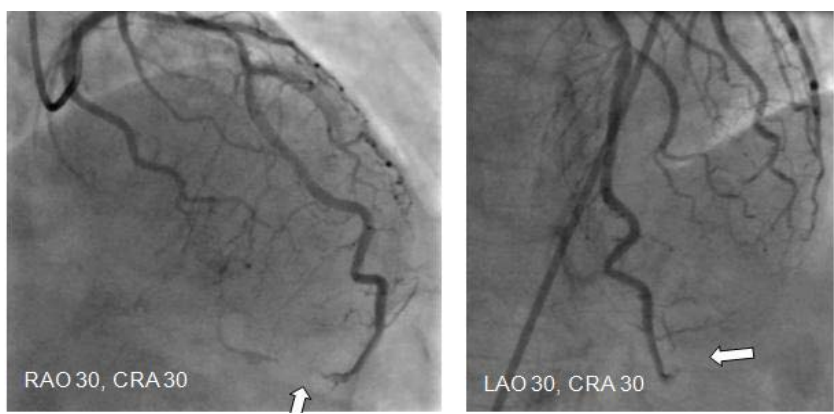

Figure 1: Filling defect in distal portion of left anterior descending (LAD) artery.
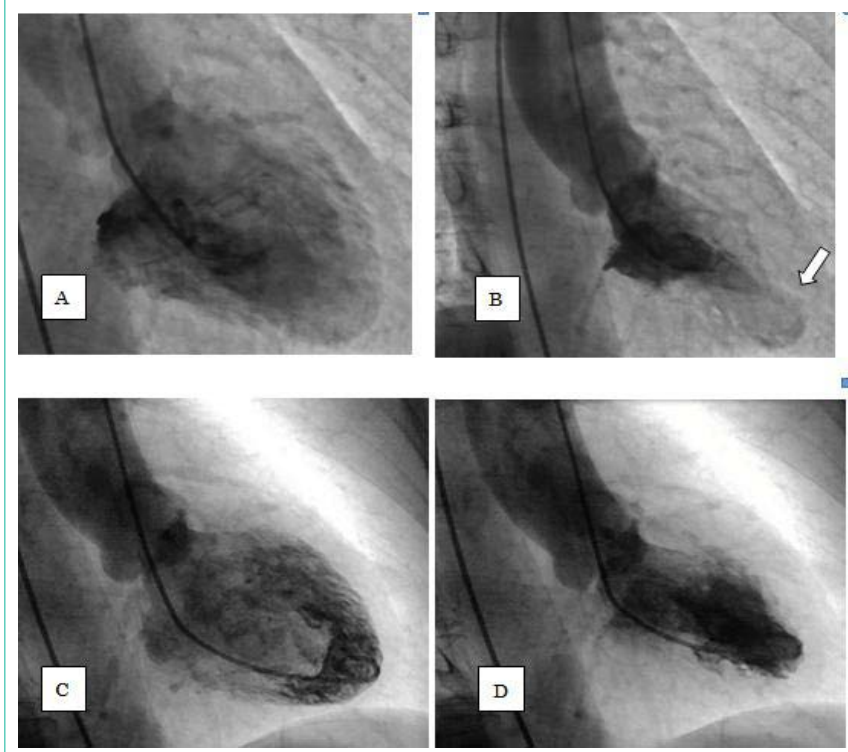

Figure 2: Left ventriculography showed hypokinesis of cardiac apex $(A, B)$, but it was improved one year. Later $(C, D)$.

stenosis. The filling defect in the distal portion of the LAD artery, which is not usually observed in the occlusion of acute coronary
Ann Hematol Oncol - Volume 4 Issue 9 - 2017

Sadahiro et al. (C) All rights are reserved
Citation: Sadahiro H, Takamichi M, Junji Y, Takamasa I, Keita W, Yuichiro S, et al. A Case of Acute Myocardial Infarction due to Coronary Spasm that Resulted in Thrombi. Ann Hematol Oncol. 2017; 4(9): 1169. 

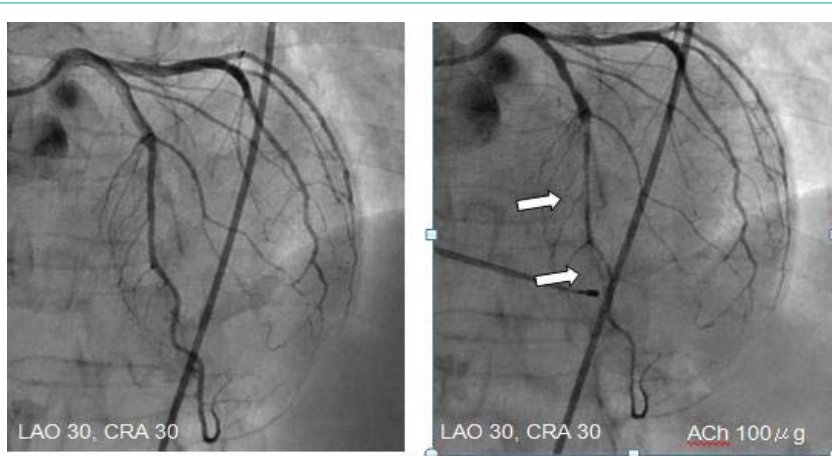

Figure 3: Acetylcholine intracoronary administration provoked coronary spasm with symptoms.

syndrome, formed gaps by accumulation of fine thrombi with a "pebble" appearance.

Hence, on the basis of the arteriosclerosis, we think that the cause of the coronary event was not plaque rupture but blood flow stasis due to the CAS. The obstruction of the coronary artery was distal and the range of the damaged myocardium was small, so we did not administer coronary artery intervention. Medication with aspirin, anticoagulants, and coronary dilators to the coronary vasospastic angina was started.

The coronary artery stenosis and filling defect were not observed on the follow-up coronary angiography after 1 year, so we presumed that the anticoagulant therapy dissolved the blood clots. The fact that acetylcholine intracoronary administration induced CAS is not a direct proof of the causal relationship between CAS and ACS but can be a strong basis to suspect an association.

As mechanisms of CAS, nitrogen monoxide (NO) production abnormality based on endothelial dysfunction and vascular smooth muscle cell hypercontraction were reported [1,2]. Oxidative-stress marker malondialdehyde-modified low-density lipoprotein (MDALDL) showed association with vasospastic angina [3]. Predictors of CAS include smoking [4], low diastolic blood pressure, and systemic low-grade inflammation [5]. In the onset of acute myocardial infarction caused by CAS, coronary artery thrombi were formed by coronary artery plaque rupture or hypercoagulability [6]. The protrusion of the plaque contents caused by the rupture of the fibrous capsule caused the thrombosis [7]. Production of fibrinopeptide A, which is the most sensitive index of platelet aggregation ability and thrombin generation in vivo after a coronary spasm attack, increases at night and in the morning, when the activity of coronary spasm attacks is high [8]. Therefore, we suggest that CAS may itself cause hypercoagulability in the coronary artery.

\section{Conclusion}

We report a case of coronary thrombus due to coronary spasm with a unique coronary angiogram finding of acute myocardial infarction. In case a coronary filling defect does not present with arteriosclerosis, a diagnosis of coronary spasm should be considered.

\section{References}

1. Yasue $H$, Nakagawa $H$, Itoh $T$, Harada E, Mizuno Y. Coronary artery spasmclinical features, diagnosis, pathogenesis, and treatment. J Cardiol. 2008; 51: 2-17.

2. Shimokawa H. European Heart Journal. 2014; 35: 3180-3193.

3. Ohmi T, Nozato T, Sakakibara A, Nomoto H, Ohno M, Takahashi $\mathrm{Y}$, et al. Malondialdehyde-Modified Low Density Lipoprotein as Oxidative-Stress Marker in Vasospastic Angina Patients. Int Heart J. 2017; 58: 335-343.

4. Ashikaga T, Nishizaki M, Fujii H, Niki S, Maeda S, Yamawake N, et al. Examination of the microcirculation damage in smokers versus nonsmokers with vasospastic angina pectoris. Am J Cardiol. 2007; 100: 962-47.

5. Sumio M, Yuji M, Eisaku H, Hitoshi N, Yoshinobu M, Yoshihiko S, et al. Differences and Interactions between Risk Factors for Coronary Spasm and Atherosclerosis - Smoking, Aging, Inflammation, and Blood Pressure. Intern Med. 2014; 53: 2663-2670.

6. Gutstein DE, Fuster V. Pathophysiology and clinical significance of atherosclerotic plaque rapture. Cardiovasc Res. 1999; 41: 323-333.

7. Richardson PD, Davies MJ, Born GV. Influence of plaque configuration and stress distribution on fissuring of coronary atherosclerotic plaques. Lancet. 1989; 2: 941-944.

8. Ogawa H, Yasue H, Oshima S, Okumura K, Matsuyama K, Obata K. Circadian variation of plasma fibrinopeptide $A$ level in patients with variant angina. Circulation. 1989; 80: 1617-1626.
Ann Hematol Oncol - Volume 4 Issue 9 - 2017

ISSN : 2375-7965 | www.austinpublishing group.com

Sadahiro et al. (@) All rights are reserved
Citation: Sadahiro H, Takamichi M, Junji Y, Takamasa I, Keita W, Yuichiro S, et al. A Case of Acute Myocardial Infarction due to Coronary Spasm that Resulted in Thrombi. Ann Hematol Oncol. 2017; 4(9): 1169. 